\title{
Mesclando práticas em Comunicação Alternativa: caso de uma criança com autismo
}

\author{
Débora Regina de Paula Nunes \\ Universidade Federal do Rio Grande do Norte - RN \\ Larissa Bezerra dos Santos \\ Universidade Federal do Rio Grande do Norte - RN
}

\begin{abstract}
Resumo
A literatura científica evidencia os efeitos promissores do uso da Comunicação Alternativa e Ampliada (CAA) por educandos com autismo. Entre os recursos utilizados da CAA por essa população destacam-se o Picture Exchange Communication System (Pecs) e as estratégias derivadas do Aided Modeling Intervention (AMI). O objetivo do presente estudo consistiu em avaliar a eficácia de uma adaptação do protocolo Pecs e das estratégias do AMI para o desenvolvimento da comunicação de uma criança autista de cinco anos, tendo-se uma professora como agente de intervenção. Os dados foram coletados durante atendimento pedagógico realizado em uma clínica. No programa de intervenção a professora foi capacitada a usar o Pecs associado a estratégias do AMI para aumentar a frequência de interações com o aluno no decorrer das atividades pedagógicas. Um delineamento quase experimental registrou aumento na frequência de iniciativas de interação do aluno com a utilização dos pictogramas e com mudanças no estilo de interação da professora.
\end{abstract}

Palavras-chave: Comunicação; criança; autismo.

\section{Merging practices in Alternative Communication: An autism case study}

\begin{abstract}
Communication impairments, typically observed in individuals with autism, may be minimized by the use of Augmentative and Alternative Communication (AAC). The Picture Exchange Communication System (PECS) and Aided Modeling Intervention (AMI) are AAC protocols typically used with this population. The aim of this study was to evaluate the effectiveness of adapting PECS for a preschooler with autism, having his teacher as an intervention agent. Data were collected during individualized teaching sessions, conducted at a private clinic. The teacher was taught to implement the PECS protocol, associated with AMI Strategies to increase the frequency of interactions with the student during educational activities. A quasi-experimental design revealed increase in the student's communication turns using the pictograms and changes in the teacher's communication style.
\end{abstract}

Key words: communication; child; autism.

\section{Mezclando prácticas en Comunicación Alternativa: caso de un niño con autismo}

\begin{abstract}
Resumen
La literatura científica evidencia los efectos promisores del uso de la Comunicación Alternativa y Ampliada (CAA) por educandos con autismo. Entre los recursos utilizados de la CAA por esa populación se destacan el Picture Exchange Communication System (Pecs) y las estrategias derivadas del Aided Modeling Intervention (AMI). El objetivo del presente estudio se constituyó en evaluar la eficacia de una adaptación del protocolo Pecs y de las estrategias del AMI al desarrollo de la comunicación de un niño autista de cinco años que tuvo una profesora como agente de intervención. Los datos fueron recolectados durante atención pedagógica realizada en una clínica. En el programa de intervención la profesora fue capacitada a usar el Pecs asociado a estrategias del AMI para aumentar la frecuencia de interacciones con el alumno en el transcurso de actividades pedagógicas. Un delineamiento casi experimental registró aumento en la frecuencia de iniciativas de interacción del alumno con la utilización de los pictogramas y con cambios en el estilo de interacción de la profesora.
\end{abstract}

Palabras-clave: comunicación; niño; autismo. 


\section{Introdução}

Os "Transtornos Invasivos do Desenvolvimento" abrangem uma gama de síndromes constituídas por distúrbios qualitativos na interação social recíproca, na comunicação verbal e não verbal e na manifestação de padrões restritos de interesses, atividades e condutas repetitivas e estereotipadas (Klin, 2006). Considerados transtornos de alta incidência que afetam um em cada 160 indivíduos (Elsabbagh, \& cols. 2012), esse conjunto de síndromes, com etiologias múltiplas, atinge homens em uma proporção quatro vezes maior do que mulheres (Klin, 2006). Dentre os transtornos que compõem os TID, o mais prevalente e conhecido é o transtorno autista ou autismo.

Atrasos significativos no desenvolvimento da linguagem constituem uma característica determinante do autismo. Embora as estimativas variem, entre 20 e 30\% dessa população não desenvolvem, para fins comunicativos, a linguagem verbal (Klin, 2006). Dos que falam, muitos evidenciam atipicidades como ecolalia, inversão pronominal ou dificuldades na prosódia (Macedo, \& Orsati, 2011).

Prejuízos na compreensão da linguagem falada é outra particularidade dessa população (Klin, 2006). Alguns estudos sugerem que essa dificuldade está relacionada às disfunções sociais inerentes à síndrome (Mongillo, Irwin, Whalen, Klaiman, \& Schultz, 2008). Esses dados são corroborados por pesquisas que indicam que pessoas com autismo têm dificuldades em compreender expressões faciais e identificar emoções em tons de voz e evidenciam preferência por estímulos não verbais em relação aos verbais (Mongillo, \& cols., 2008).

Por seus prejuízos na comunicação expressiva e receptiva, pessoas com autismo são consideradas boas candidatas ao uso da Comunicação Alternativa e Ampliada (Nunes, 2008; Wendt, 2009; Macedo, \& Orsati, 2011). A CAA é uma área da prática clínica e educacional que objetiva compensar, temporária ou permanentemente, os prejuízos na comunicação expressiva e receptiva (Beukelman, \& Mirenda, 1998). A CAA envolve o uso de gestos manuais, expressões faciais e corporais, símbolos gráficos (fotografias, gravuras, desenhos, linguagem alfabética, objetos reais e miniaturas), voz digitalizada ou sintetizada e outros meios de efetuar a comunicação face a face de pessoas que apresentam limitações no uso e/ou compreensão da linguagem oral. Os recursos da CAA podem substituir ou suplementar as funções da fala. A denominada "Comunicação Alternativa" se dá quando os recursos substituem a fala, e a Comunicação Ampliada, quando a suplementam.

O uso de sistemas alternativos de comunicação para pessoas com autismo vem sendo registrado na literatura desde a década de 1970 (Nunes, 2008). A partir desse período, três tipos de sistemas têm se destacado: sinais manuais, sistemas pictográficos assistidos de baixa tecnologia e sistemas assistidos com acionadores de voz (para revisão ver Nunes, 2008; Wendt, 2009). Entre os sistemas pictográficos reportados merecem destaque os protocolos que empregam estratégias derivadas do Aided Modeling Intervention (AMI) e o Picture Exchange Communication System (Pecs).

O AMI é um modelo de intervenção que prevê o uso de sistemas assistidos de CAA por parceiros sociais como forma de promover a comunicação de pessoas desprovidas de fala funcional (Drager, 2009). Pesquisas indicam que o uso do sistema pelos parceiros pode reduzir comportamentos inadequados (Drager, \& cols., 2006), assim como favorecer a compreensão de enunciados verbais e estimular o uso da CAA por pessoas autistas (Cafiero, 2001; Drager, \& cols., 2006). O AMI adota estratégias derivadas do Ensino Naturalístico (EN), um modelo geral de intervenção em linguagem que valoriza a dimensão pragmática da linguagem, mesclando pressupostos teóricos do modelo comportamental com teorias interacionistas do desenvolvimento infantil, além de outras proposições (Nunes, 1992; Drager, 2009). O EN, que ganhou notoriedade a partir da década de 1980, surgiu como resposta às dificuldades evidenciadas nas estratégias de ensino derivadas do modelo comportamental em promover a generalização de respostas aprendidas em ambientes artificiais (Prizant, \& Wetherby, 1998). Na atualidade, o EN é compreendido como uma prática cientificamente válida para populações com autismo (National Autism Center, 2011). Entre os elementos característicos desse modelo destacam-se: a) o envolvimento de parceiros sociais significativos (familiares, pessoas próximas, amigos); b) o uso de reforçadores indicados pelo educando; c) o uso de contingências do meio natural como mecanismo de aumentar a frequência de emissões de comportamentos desejadas; e e) o ensino realizado durante atividades cotidianas em contextos e ambientes naturais (Drager, 2009; Nunes, 1992; Prizant, \& Wetherby, 1998).

O Pecs ou Sistema de Comunicação por Troca de Figuras foi desenvolvido por Bondy e Frost (1994) em resposta às dificuldades de uma parcela expressiva de pacientes com autismo em desenvolver a comunicação funcional por meio de treino verbal ou o uso da língua de sinais (Nunes, 2008). É um protocolo de ensino desenvolvido em seis fases que capacita o indivíduo com distúrbios de comunicação a se expressar por meio de um sistema pictográfico. De forma específica, ele aprende que, entregando cartões contendo representações visuais de objetos/ações almejadas consegue ter acesso a esses itens. O programa tem seus pressupostos teóricos apoiados na Análise Experimental do Comportamento, que considera a linguagem como um comportamento como qualquer outro, adquirido a partir de contingências (Frost, \& Bondy, 2002). O contexto estruturado e o uso de estímulos concretos (figuras) proposto pelo Pecs parecem favorecer a rápida aprendizagem do sistema (Fidalgo, Godoi, \& Gioia, 2008).

Em uma das primeiras investigações sobre o Pecs, Bondy e Frost (1994) avaliaram os efeitos do referido protocolo nas habilidades comunicativas de 85 crianças com TID. Os resultados indicaram que uma parcela expressiva dos participantes aprendeu a utilizar os pictogramas, associados ou não à linguagem verbal. 
Desde a publicação da pesquisa de Bondy e Frost (1994), diversos estudos replicando o Pecs foram produzidos. Muitas dessas investigações foram sumarizadas em revisões científicas publicadas na literatura nos últimos anos (Tien, 2008; Sulzer-Azaroff, Hoffman, Horton, Bondy, \& Frost, 2009; Preston, \& Carter, 2009; Flippin, Reska, \& Watson, 2010). Tien (2008) sintetizou os achados de treze estudos desse tipo, envolvendo 125 participantes com autismo, publicados entre 1998 e 2005. Sulzer-Azaroff e colaboradores (2009), por sua vez, analisaram os efeitos do Pecs em autistas em 23 pesquisas produzidas entre 1996 e 2007. Preston e Carter (2009) ampliaram essas análises, avaliando os efeitos do Pecs em 456 participantes, distribuídos em 27 estudos publicados entre 1993 e 2007 . Por fim, Flippin e colaboradores (2010) avaliaram artigos publicados entre 1994 e 2009. Essas revisões científicas indicaram que o Pecs favoreceu a comunicação de uma parcela expressiva de pessoas com TID, com limitada fala funcional.

Contrapondo-se aos achados de Bondy e Frost (1994), o uso do sistema nem sempre resultou no desenvolvimento da oralidade dos participantes autistas (Preston, \& Carter, 2009; Flippin, \& clos., 2010). Da mesma forma, os efeitos do Pecs na redução de comportamentos desadaptativos não foram consistentes em diversos estudos analisados (Preston, \& Carter, 2009). Assim, embora algumas revisões sugiram que o Pecs é uma prática empiricamente validada ${ }^{1}$ (Tien, 2008; Sulzer-Azaroff, \& cols., 2009), outros estudos salientam a necessidade de mais pesquisas (Flippin, \& cols., 2010).

Adaptações do sistema Pecs (e.g. Cummings, \& Williams, 2000; Kravits, Kamps, Kemmerer, \& Potucek, 2002), assim como outros protocolos que empregam a comunicação por meio de trocas de figuras, têm sido registradas na literatura internacional e evidenciado resultados promissores (Frea, Arnold, \& Vittimberga, 2001; Nunes, \& Hanline, 2007). Cummings e Williams (2000) - por exemplo, combinaram o Pecs com treino verbal para desenvolver em crianças autistas (três a cinco anos) a comunicação expressiva. Os resultados da pesquisa indicaram que quatro dos cinco participantes aprenderam a usar o Pecs e a verbalizar o que ouviam. No estudo de Kravits e colaboradores (2002) o Pecs foi associado ao treino de habilidades sociais em uma menina autista de seis anos. Os dados revelaram que é adequado o uso do sistema pictográfico e que aumentou a frequência de interações sociais da criança nos contextos escolar e familiar.

Frea e colaboradores (2001), por sua vez, utilizaram um sistema de comunicação por meio de troca de figuras, para reduzir os comportamentos desadaptativos de um menino não verbal de quatro anos com diagnóstico de autismo. Através de estratégias comportamentais, a crian-

1 Práticas cientificamente válidas são definidas como métodos e técnicas que tenham satisfeito padrões científicos rigorosos determinados por múltiplos revisores e que, quando replicados de forma consistente, válida e fidedigna, tenham produzido resultados positivos (Nunes, Nunes Sobrinho, 2010). ça aprendeu a solicitar itens de sua preferência por meio dos pictogramas. Essa habilidade resultou na redução de comportamentos agressivos. Esses dados corroboram os achados de Bondy e Frost (1994) sobre a redução de comportamentos agressivos mediante a aprendizagem de um sistema de CAA.

Um sistema de comunicação por meio de troca de figuras, não caracterizado como o Pecs, foi também utilizado em estudo conduzido por Nunes e Hanline (2007). As referidas autoras capacitaram um pai a ensinar o seu filho autista (quatro anos) a utilizar os pictogramas em duas rotinas domésticas: alimentação e brincadeira. No programa de capacitação, o pai aprendeu a empregar estratégias naturalísticas de ensino para promover o uso do sistema de comunicação. Os resultados da pesquisa revelaram que as estratégias ensinadas ao pai promoveram o aumento nos turnos de comunicação e no uso dos pictogramas pelo filho.

No Brasil é incipiente o número de estudos publicados em periódicos científicos sobre o uso do Pecs ou outros protocolos que ensinem a comunicação expressiva ou receptiva por meio de trocas de figuras para pessoas com autismo, apenas identificamos o de Walter e Almeida (2010) e o de Ferreira, Teixeira e Brito (2011). No estudo de Walter e Almeida (2010), três mães foram capacitadas a aplicar, utilizar e manter o uso da CAA no ambiente familiar com filhos adolescentes autistas. A CAA empregada foi baseada no Pecs-adaptado, um programa fundamentado no Currículo Funcional Natural, ${ }^{2}$ que utiliza o intercâmbio de figuras em situações naturais. Através de um delineamento quase experimental de múltiplas sondagens, foram avaliados os efeitos da capacitação nos comportamentos das três díades (mãe-filho). Assim como no estudo de Frea e colaboradores (2001), Walter e Almeida (2010) registraram nos adolescentes investigados redução de comportamentos indesejados após a introdução do sistema. Da mesma forma, foi observado aumento na frequência de atos comunicativos dos filhos e melhor clareza nas situações comunicativas das díades.

O Pecs-adaptado foi igualmente utilizado na investigação conduzida por Ferreira e colaboradores (2011). Esse estudo teve por objetivo descrever os efeitos da utilização simultânea de dois métodos alternativos de comunicação na ampliação das habilidades pragmáticas de um adulto com autismo. Os métodos incluíam o Pecs-adaptado, já descrito, e o uso da fala sinalizada (uso simultâneo da fala e de um gesto correspondente). Os resultados indicaram aumento no número de atos comunicativos e funções comunicativas empregadas pelo participante.

As pesquisas acima assinaladas revelam resultados promissores provindos do uso de protocolos que preveem a utilização de sistemas assistidos por parceiros sociais (como os derivados do AMI), assim como programas que empregam mecanismos de troca de figuras, como o Pecs ou

2 O CFN fundamenta-se numa filosofia de educação que determina a forma e o conteúdo de um currículo adequado às características individuais. Requer uma metodologia instrucional que enfatiza a aplicação do conhecimento e habilidades em contexto real (Miura, 2008, p. 155). 
adaptações desse sistema para pessoas com autismo. As distintas metodologias de ensino empregadas nesses programas, embora advenham de abordagens diferentes (ex.: abordagem comportamental ou naturalista), parecem ser efetivas para ensinar a CAA para essa população. Conforme sinaliza Thompson (2011), enquanto os procedimentos comportamentais são considerados eficazes para instalar um comportamento, os derivados da abordagem naturalística são considerados efetivos para promover sua generalização.

Nesse cenário, o presente estudo tem como propósito avaliar a eficácia do Pecs associado a estratégias naturalísticas de ensino derivadas do AMI no desenvolvimento da comunicação de uma criança de cinco anos com diagnóstico de autismo, tendo uma professora como agente de intervenção.

\section{Método}

\section{Participantes}

A presente pesquisa foi conduzida com Pedro, um aluno com diagnóstico de autismo, e Neusa, sua professora de apoio pedagógico. Pedro tinha cinco anos e estava matriculado no nível IV da Educação Infantil. Aos três anos recebera diagnóstico de autismo, por um neuropediatra. No início da pesquisa, o grau de autismo de Pedro foi considerado moderado por meio do instrumento Childhood Autism Rating Scale - Cars (Pereira, Riesgo, \& Wagner, 2008), aplicado pelas autoras. Pedro não se comunicava verbalmente, mas empregava gestos idiossincráticos para expressar suas vontades. Verbalizava, por vezes, palavras fora de contexto e apresentava adequada compreensão da fala. Exibia comportamentos ritualísticos, estereotipias motoras, inflexibilidade comportamental e severa dificuldade em interagir com seus pares.

Neusa, 38 anos, era formada em Pedagogia e trabaIhava com alunos com TID e outros transtornos do desenvolvimento havia um ano. Sempre atuara como auxiliar de sala na Educação Infantil em escolas privadas. No período em que a pesquisa foi conduzida, estava trabalhando em uma sala de apoio pedagógico alocada em uma clínica particular.

\section{Local}

O estudo foi desenvolvido em uma sala de atendimento pedagógico individualizado em uma clínica particular situada na cidade de Natal, RN.

\section{Materiais}

Foram utilizados os seguintes materiais: 1- uma filmadora digital; 2- uma câmera fotográfica; 3- Pictogramas; 4- Jogos; 5- atividades pedagógicas realizadas em folha; 6o Manual de Treinamento do Sistema de Comunicação por
Troca de Figuras (The Picture Exchange Communications System. Training Manual) (Frost, \& Bondy, 2002).

\section{Instrumentos}

No estudo foi utilizado um diário de campo, onde foram registradas as impressões e anotações da pesquisadora (segunda autora).

\section{Programa de capacitação}

No programa de capacitação, a professora foi ensinada a empregar as três primeiras fases do Pecs e duas estratégias naturalísticas derivadas do modelo AMI durante atividades pedagógicas. Conforme orientação do Manual Pecs (Frost, \& Bondy, 2002), a primeira fase do sistema consiste em ensinar o aluno a trocar o pictograma de um objeto por seu referente, com a ajuda de dois treinadores: um deles (parceiro de comunicação) posiciona-se na frente da criança, e o outro, denominado estimulador físico, permanece atrás dela. Um cartão, com a ilustração (foto ou desenho) de um item altamente motivador para a criança, é posicionado entre ela e o parceiro de comunicação. O parceiro, então, segura o item representado no pictograma e olha para a criança. Se a criança tentar pegar o item, o estimulador físico a ajuda a buscar o pictograma e entregá-lo ao parceiro de comunicação. Após a entrega do pictograma, o item almejado é entregue ao aluno.

Na segunda fase, cujo objetivo é promover a comunicação espontânea, o aluno é ensinado a mover-se em direção à prancha de comunicação, selecionar o pictograma desejado e entregá-lo ao parceiro de comunicação, de forma independente. Na terceira fase, caracterizada como etapa de discriminação, são apresentadas simultaneamente figuras de itens desejados e de itens irrelevantes. O objetivo é fazer com que a criança distinga os pictogramas e entregue ao parceiro o cartão correspondente ao elemento desejado e apropriado ao contexto de interação.

Concomitantemente ao treinamento do Pecs, a professora foi instruída a utilizar estratégias naturalísticas de ensino, contemplando o uso da CAA, conforme preconizado pelo modelo do AMI. De forma específica, foram apresentadas duas estratégias anteriormente utilizadas em estudos conduzidos por Nunes e Hanline (2007), Gomes (2011) e Nunes, Azevedo e Freire (2011): o arranjo ambiental e o mando com CAA. O quadro 1, abaixo, define essas estratégias:

Variáveis do aluno - as respostas do aluno são categorizadas em atendimento a comandos, tipos de turno e modalidades de respostas empregadas nos turnos. $\mathrm{O}$ atendimento a comandos diz respeito a comportamentos não comunicativos emitidos pelo aluno após instruções verbais do professor. Eles referem-se, por exemplo, a respostas de sentar após o comando "sente-se", ou a abrir uma porta após o comando "abra a porta". Esses comportamentos, que avaliam a compreensão de enunciados verbais, foram 
Quadro 1. Estratégias Naturalísticas de Ensino utilizadas no estudo.

\begin{tabular}{ll}
\hline Estratégias & Definição \\
\hline $\begin{array}{l}\text { Arranjo } \\
\text { ambiental }\end{array}$ & $\begin{array}{l}\text { Caracteriza-se pela organização física do ambiente, onde os pictogramas são postos próximos ao } \\
\text { aluno e objetos de seu interesse dispostos em seu campo visual, mas fora de seu alcance. }\end{array}$ \\
\hline Mando com CAA & $\begin{array}{l}\text { A professora tece comentários, formula perguntas e faz solicitações utilizando simultaneamente a } \\
\text { linguagem oral e o sistema pictográfico de comunicação. }\end{array}$ \\
\hline
\end{tabular}

Quadro 2. Tipos de turnos do aluno

\begin{tabular}{cl}
\hline Turno & Definição \\
\hline Resposta & $\begin{array}{l}\text { Enunciados verbais/vocais, gestuais ou pictográficos emitidos pelo aluno após pergunta/solicitação } \\
\text { do professor. }\end{array}$ \\
Iniciativa & $\begin{array}{l}\text { Enunciados verbais/vocais, gestuais (gestos, língua de sinais e expressões faciais) ou pictográficos } \\
\text { emitidos pelo aluno/professora sem ocorrência de solicitação de resposta do parceiro. }\end{array}$ \\
\hline
\end{tabular}

Quadro 3: Modalidade de respostas nos turnos.

\begin{tabular}{ll}
\hline \multicolumn{1}{c}{ Modalidade } & Definição \\
\hline Gestual & $\begin{array}{l}\text { Ação gestual (convencional; simbólica e de contenção) emitida pelo aluno durante um turno } \\
\text { (iniciativa ou resposta). }\end{array}$ \\
Verbal/vocal & $\begin{array}{l}\text { Verbalizações, sons guturais, gemidos, murmúrios, balbucios ou vocábulos não inteligíveis } \\
\text { emitidos pelo aluno durante um turno (iniciativa ou resposta). }\end{array}$ \\
Pictográfica & $\begin{array}{l}\text { Uso de pictograma (fichas com fotos da rotina e objetos do aluno dispostas na mesa, ou numa } \\
\text { pasta de comunicação) pelo aluno durante um turno (iniciativa ou resposta). }\end{array}$ \\
\hline
\end{tabular}

registrados à medida que o aluno acatava as instruções verbalizadas pela professora.

As variáveis tipos de turnos e modalidades de respostas foram derivadas de estudos realizados anteriormente por Nunes e Hanline (2007), Gomes (2011) e Nunes, Azevedo e Freire (2011). Turnos são definidos como enunciados emitidos pelo aluno de forma espontânea ou após solicitação do professor. O quadro 2, acima, contempla a definição dos dois tipos de turnos analisados no presente estudo.

Os turnos (iniciativa e resposta) foram posteriormente categorizados em três modalidades, conforme indica o quadro 3 , acima.

\section{Variáveis da professora}

No repertório da professora foram avaliadas as variáveis uso das duas estratégias naturalísticas de ensino previamente descritas (arranjo ambiental e mando com CAA) e frequência de mandos. O mando é definido como perguntas ou solicitações orais da professora dirigidas ao aluno. O propósito da inserção dessa variável foi avaliar se o aluno respondia aos comandos da professora quando ela não utilizava os pictogramas.

\section{Procedimentos}

O referido estudo está inserido em um projeto intitulado "Análise dos Efeitos de um Plano Individualizado de Educação no Desenvolvimento Acadêmico de um Aluno Autista", submetido ao Comitê de Ética em Pesquisa da Universidade Federal do Rio Grande do Norte e por ele aprovado mediante o Parecer no 63.139. Após a aprovação do projeto pelo Comitê de Ética, o estudo foi conduzido em três etapas: linha de base, capacitação e intervenção.

\section{Etapa 1: Linha de base}

Nesta fase foram feitos registros fotográficos dos materiais utilizados por Neusa durante o trabalho com Pe- 
dro. Adicionalmente, a segunda autora instruiu a professora a utilizar livremente os pictogramas ${ }^{3}$ disponíveis na sala durante as atividades pedagógicas realizadas com o aluno. As sessões foram videogravadas pela segunda autora, e as variáveis do aluno (atendimento a comandos, turnos e modalidade de turnos), assim como as da professora (arranjo ambiental, mando e mando com CAA), foram analisadas. Com a finalidade de manter a homogeneidade das sessões, foram categorizados apenas os primeiros três minutos de interação da díade.

\section{Etapa 2: Capacitação}

Esta etapa teve início quando foi evidenciada, por meio de inspeção visual, estabilidade na frequência de turnos comunicativos do aluno durante a realização de atividades pedagógicas. Neusa foi instruída pela segunda autora sobre como utilizar o sistema Pecs associado às estratégias naturalísticas de ensino. Optou-se por realizar essa capacitação durante uma nova atividade, a rotina de lanche. A capacitação ocorreu na mesma sala em que Neusa atendia Pedro, e foi realizada pela segunda autora. As orientações duravam, em média, trinta minutos, e ocorriam por meio de exposições orais, dramatizações e treinamento com a criança. Como o aluno não utilizava o sistema de forma espontânea, a segunda autora atuou como estimulador físico. Vale ressaltar que essa etapa da intervenção durou dois meses e não foi videogravada nem considerada nas análises.

Etapa 3: Intervenção. Esta fase teve início quando o aluno evidenciou domínio no uso do sistema, especificamente durante a fase 3 do protocolo Pecs. Nesse momento, Neusa foi instruída a utilizar o sistema Pecs associado às duas estratégias naturalísticas durante as atividades pedagógicas. O propósito era que o uso do sistema pelo aluno fosse generalizado para outro contexto (atividade pedagógica) e mantido por meio das estratégias. Nessa fase, a pesquisadora limitava-se a videogravar as sessões, não fornecendo dicas ou orientações à professora durante a atividade. Quando a câmera era desligada, a pesquisadora e a professora conversavam sobre a atividade realizada, identificando os pontos positivos e negativos da sessão. Nesses episódios, a pesquisadora ressaltava o uso das estratégias aprendidas durante a capacitação na rotina do lanche. Assim como nas sessões de linha de base, foram categorizadas as respostas (variáveis do aluno e da professora) dos primeiros três minutos de interação da díade no decorrer da realização das atividades pedagógicas.

\section{Medidas de fidedignidade}

As sessões foram randomicamente codificadas por dois assistentes de pesquisa, que receberam treinamento antes do início do estudo. Com o objetivo de avaliar o índice

3 Os pictogramas foram elaborados por Neusa e estavam disponíveis na sala antes do início da pesquisa. de fidedignidade das categorias analisadas, a segunda autora codificou $25 \%$ das sessões, aleatoriamente selecionados. O cálculo de concordância variou entre 70 e 95\%, indicando adequada fidedignidade, de acordo com Fagundes (1985).

\section{Delineamento de pesquisa}

Um delineamento quase experimental do tipo A-B linha de base - intervenção (Kennedy, 2005) foi empregado para avaliar os efeitos do programa de intervenção nas respostas da díade. No total foram realizadas vinte e quatro sessões (onze de linha de base e treze de intervenção) no decorrer de seis meses.

\section{Resultados}

A presente pesquisa teve como propósito avaliar a eficácia do Pecs associado a estratégias naturalísticas de ensino derivadas do AMI no desenvolvimento da comunicação de uma criança de cinco anos com diagnóstico de autismo, tendo uma professora como agente de intervenção. Inicialmente buscou-se identificar os efeitos do programa de capacitação nas respostas da professora. Em seguida, o propósito foi avaliar os efeitos das estratégias de ensino empregadas pela professora no comportamento comunicativo do aluno. Os resultados, abaixo descritos, foram produzidos com base nos diários de campo e na análise das sessões videogravadas.

Conforme observado na figura 1 , o programa de capacitação parece não ter afetado o estilo de interação verbal da professora com Pedro. De forma específica, o uso da linguagem oral (mando) foi predominante tanto na fase de linha de base quanto na de intervenção. $O$ uso dos pictogramas associado a comandos verbais foi registrado no repertório de Neusa em apenas um episódio da fase de linha de base, e não foi empregado após a capacitação.

O uso do sistema de CAA pelo parceiro comunicativo, configurado pela estratégia do mando com CAA, é importante para que o aluno aprenda a usar os pictogramas para se

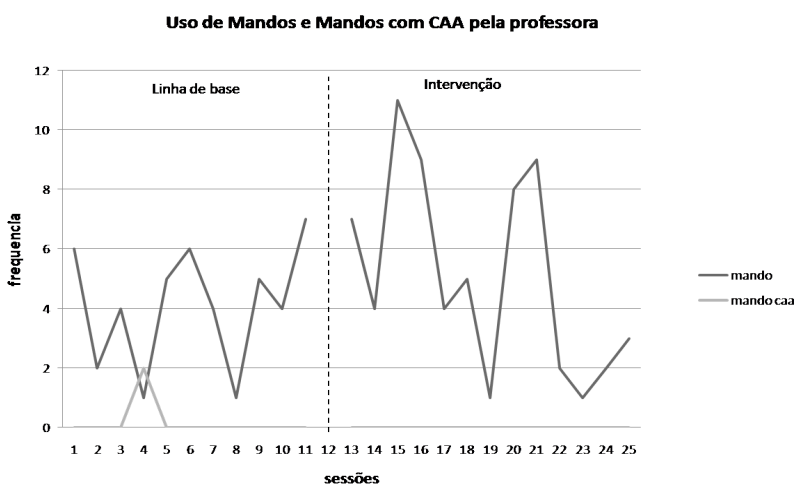

Figura 1. Frequência no uso de mandos e mandos com CAA pela professora. 
comunicar (Nunes, \& cols., 2011; Drager, 2009). O emprego desses recursos visuais pelo interlocutor também ajuda crianças com limitada compreensão verbal a compreender a fala, conforme preconiza o AMI (Drager, 2009). No presente estudo, Neusa, possivelmente, falhou em utilizar os pictogramas para se comunicar com Pedro, por duas razões: 1- conjetura-se que o aluno já havia aprendido a utilizar o sistema na segunda etapa do estudo (capacitação), não necessitando de novos modelos; e 2- o menino aparentava ter boa compreensão de enunciados verbais, por isso o uso das figuras pareceu dispensável para auxiliar na compreensão de Pedro, que parecia assentir às instruções verbais da professora, como revela a figura 2.

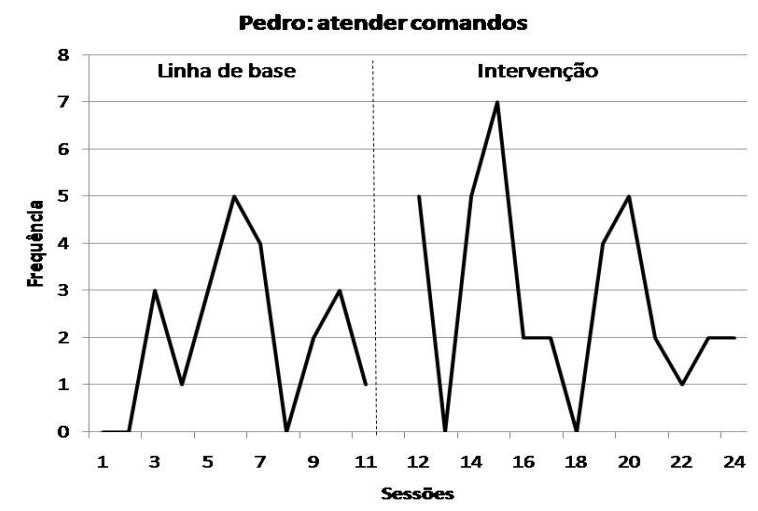

Figura 2. Frequência de atendimento a comandos de Pedro

Em outras palavras: como a professora utilizava os pictogramas com baixa frequência em suas verbalizações, é plausível pensar que o aluno apresentava boa compreensão da fala, sem necessitar da ajuda dos pictogramas (mandos com CAA).

Assim, o programa de capacitação parece não ter produzido mudanças nas formas de expressão de Neusa; porém foi observado aumento na frequência de oportunidades de comunicação ofertadas ao aluno após a capacitação. Conforme se observa na figura 3, na fase de intervenção a professora passou a usar sistematicamente as estratégias de arranjo ambiental. Neste sentido, era comum observá-la posicionando os materiais pedagógicos (tinta, lápis etc.) longe do alcance de Pedro, mas dentro de seu campo de visão. Essa estratégia é indicada tanto no protocolo Pecs quanto no modelo AMI. Conforme preconiza a literatura, o uso do arranjo ambiental incita a criança a aumentar a frequência de iniciativas de interação.

De fato, após a capacitação foi observado no repertório de Pedro um aumento na frequência de iniciativas, conforme revela a figura 4 , abaixo.

Assim, a ausência de iniciativas de interação durante a fase de linha de base pode estar relacionada às escassas oportunidades de interação ofertadas pela professora; porém essa mudança não foi observada na frequência de respostas comunicativas de Pedro. Conjetura-se que a pro-

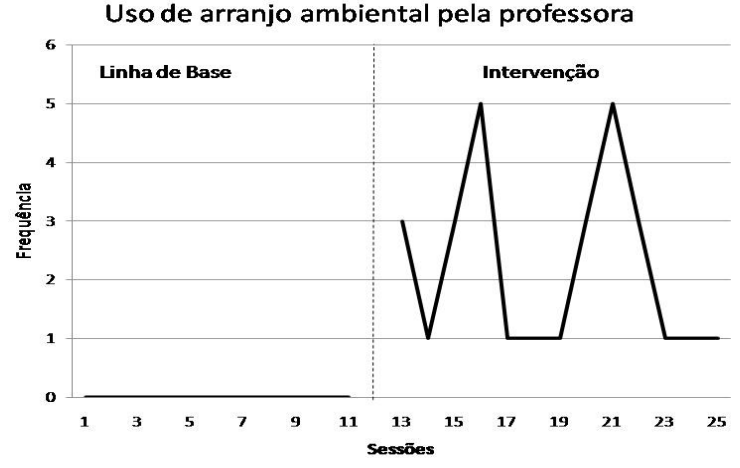

Figura 3. Frequência no uso da estratégia de arranjo ambiental pela professora

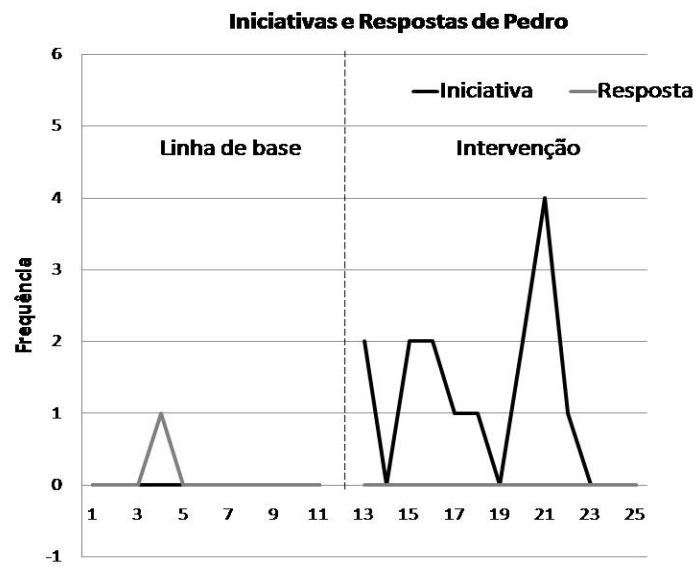

Figura 4. Frequência de iniciativas e respostas de Pedro

fessora tenha falhado em oferecer oportunidades para que o menino respondesse a perguntas específicas.

As modalidades de respostas empregadas nos turnos (iniciativas e respostas) do aluno durante as interações com a professora estão apresentadas na figura 5, abaixo.

De modo geral, os dados revelam que durante a fase de linha de base o aluno usou pouco as três modalidades de comunicação (verbal, gestual e pictográfica). Observa-se apenas um episódio em que o aluno respondeu, de forma expressiva, a um comando da professora (sessão 4 - linha de base). De acordo com os registros de campo, a resposta foi vocal:

Ao ser instruído a lavar as mãos, Pedro vocalizou "Êe", enquanto dirigia o olhar para a professora, e caminhou até o banheiro. (Registro de campo, 23/11/2011)

O pouco uso dos pictogramas pela díade na fase de linha de base, evidenciado nas figuras 1 (mando com CAA pela professora) e 5 (CAA do aluno), pode ser atribuído à forma como os cartões eram disponibilizados na sala de aula. 


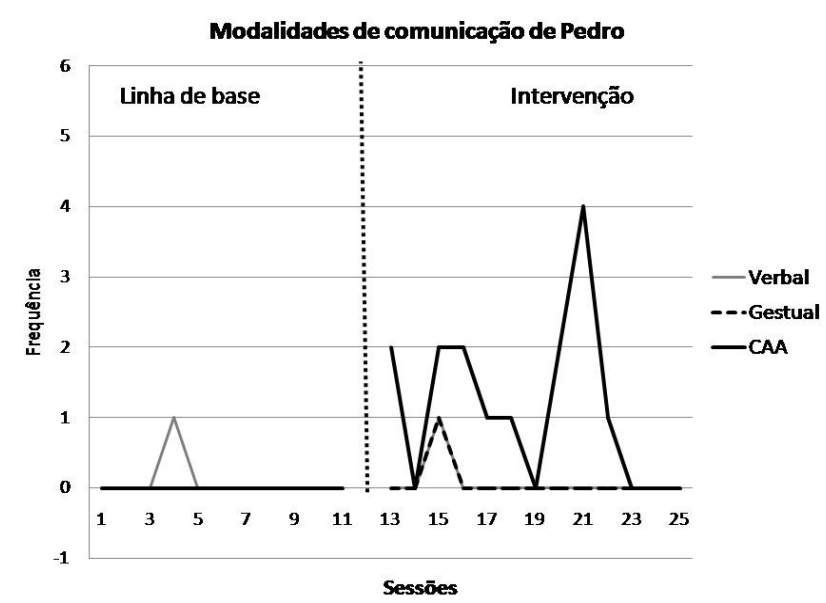

Figura 5. Frequência no uso de verbalizações/vocalizações, gestos e CAA (pictogramas) nos turnos comunicativos (iniciativas e respostas) de Pedro.

Em outras palavras, antes da capacitação, os pictogramas confeccionados por Neusa pareciam servir, primordialmente, para a visualização de rotinas na sala, não abrangendo "propósito comunicativo". Conforme se observa na figura 6 , abaixo, os pictogramas eram organizados de acordo com a sequência em que as atividade pedagógicas eram encaminhadas.

Neusa fornecia poucas oportunidades para que os pictogramas disponibilizados no quadro da rotina servissem para Pedro se comunicar com ela. De acordo com registros de campo, o único momento em que Neusa solicitou que o aluno utilizasse as figuras para se comunicar foi quando the pediu que cumprimentasse a pesquisadora com o cartão do "bom-dia". Nesse episódio, o menino manuseou o cartão de forma estereotipada, sem evidenciar interação social, conforme está assinalado no diário de campo da pesquisadora.

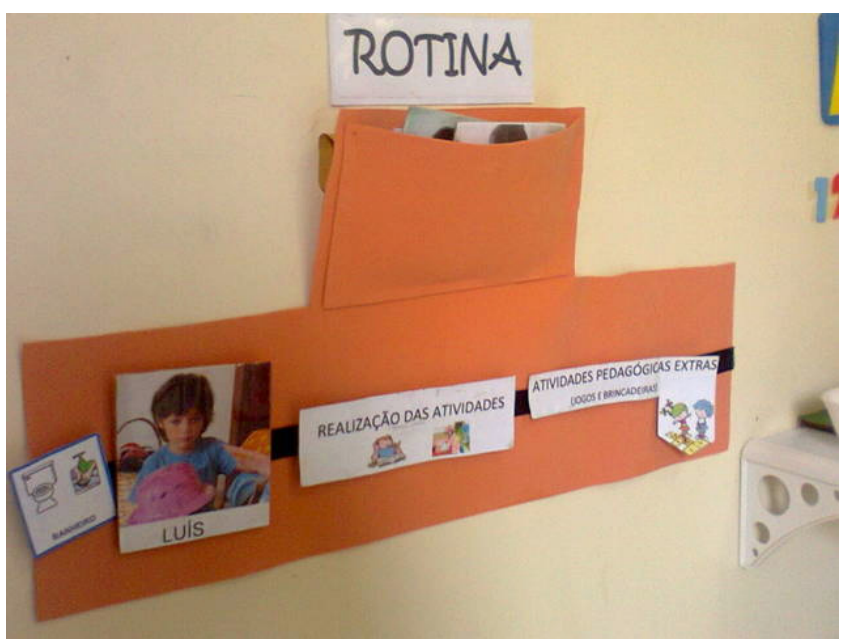

Figura 6. Quadro de rotinas de trabalho de Pedro
Pedro estava sentado no tapete colorido que ficava em frente à janela. Neusa olhava pela janela investigando como estava o dia (sol/chuva) e cantando a música "A janelinha abre quando está chovendo, a janelinha fecha quando o sol está aparecendo". Quando cheguei, ele estava com o cartão do "sol", com as palavras "Bom dia" escritas. Neusa pediu que me desse "bom-dia!", apontando para o cartão. Pedro fez movimentos estereotipados com o cartão em sua frente, mas não o entregou. (Registro de campo, 9/11/2011).

De acordo com Neusa, o único pictograma utilizado pelo aluno com propósito comunicativo era o cartão do banheiro. De fato, o registro de campo revelou que Pedro usou esse pictograma de forma independente. O menino, usualmente, entregava o cartão à professora ou o incluía na rotina quando desejava ir ao banheiro. Vale ressaltar que isso ocorria sem solicitação prévia da professora.

Após a intervenção, novos pictogramas passaram a ser utilizados com propósito comunicativo, em outros contextos. Durante as atividades pedagógicas, por exemplo, Pedro passou a utilizar os cartões para solicitar materiais como lápis, cola ou tinta colorida. Após a realização das tarefas, ordinariamente solicitava brincar com jogos, entregando os pictogramas correspondentes a Neusa.

Registros de campo indicaram que, após cada atividade, Neusa pedia a Pedro que retirasse do quadro de rotinas o cartão da atividade finalizada. Quando o último cartão referia-se à atividade livre, ela perguntava ao menino o que ele queria fazer. Nesses episódios ela apontava para os cartões disponíveis (ex.: carrinhos de brinquedo, bolhas de sabão, jogos de encaixe) enquanto verbalizava a pergunta. Pedro, então, selecionava o cartão e o entregava a ela, que, por sua vez, entregava a ele o brinquedo desejado.

É importante salientar que a intervenção impactou a frequência de iniciativas de comunicação utilizando os pictogramas, mas não foram observadas mudanças no uso de verbalizações/vocalizações de Pedro. Esses dados corroboram os achados de Ganz, Simpson e Corbin-Newsome (2007), que revelam, em seu estudo, que o Pecs não produziu efeitos na linguagem oral de alguns dos participantes com autismo.

\section{Conclusões}

A literatura científica evidencia resultados promissores obtidos com o uso dos recursos da CAA para populações que não desenvolvem a fala funcional, como os autistas. Os dados da presente pesquisa sugerem que a mera exposição desses indivíduos aos recursos da CAA pode ser pouco eficaz para o desenvolvimento de novas formas de expressão. Apesar da presença dos pictogramas em sala de aula, foram escassas as ocasiões em que Pedro utilizou os cartões para comunicar seus desejos durante a fase de linha de base. A professora, por sua vez, antes da intervenção, empregava os pictogramas essencialmente para ilustrar as rotinas em sala de aula. Eram poucas as oportunidades ofertadas ao 
aluno para usar os pictogramas como forma de comunicação expressiva. Nesse contexto, a pesquisadora propôs a Neusa o programa de capacitação no uso da Comunicação Alternativa por meio do Pecs e das estratégias naturalísticas derivadas do modelo AMI.

Durante a intervenção, mudanças foram observadas no comportamento de Neusa, que começou a fornecer ferramentas para que o aluno se comunicasse com ela. Assim, passou a organizar o ambiente de formas distintas durante as atividades pedagógicas, de maneira a incitar as iniciativas de interação do menino. Essas mudanças produziram efeitos positivos em Pedro, que se tornou mais responsivo. Adicionalmente, com a apresentação da CAA, ele, que não utilizava a linguagem oral, passou a se comunicar com ela por meio dos pictogramas.

Os resultados promissores do presente estudo podem ser atribuídos a três fatores: primeiramente, à adoção de práticas como o protocolo Pecs e o Ensino Naturalístico, que evidenciam adequado respaldo empírico (Simpson, 2005); em segundo lugar, ao delineamento de pesquisa (AB) adotado, que permite registro e monitoramento contínuos de respostas dos participantes e, por fim, ressalta-se a capacitação in loco, em que o pesquisador ensina o professor a implementar as estratégias de ensino no ambiente no qual ocorrem as interações. Essa prática auxilia na aprendizagem do professor, que pode esclarecer dúvidas no contexto de sua atuação com o aluno. De acordo com Nunes (2008), a colaboração entre professores e educadores favorece a implementação de práticas pedagógicas empiricamente validadas.

\section{Referências}

Beukelman, D., \& Mirenda, P. (1998). Augmentative and alternative communication: Management of severe communication disorders in children and adults. Second Edition. London, UK: Paul $\mathrm{H}$. Brookes.

Bondy, A. S., \& Frost, L. A. (1994). The picture exchange communication system. Focus on Autistic Behavior, 9(3), 1-19.

Cafiero, J. (2001). The effect of an augmentative communication intervention on the communication, behavior and academic progress of an adolescent with autism. Focus on Autism and Other Developmental Disabilities, 16 (3), 179-189.

Cummings, A. R., \& Williams, W. L. (2000). Visual identity matching and vocal imitation training withchildren with autism: A surprising finding. Journal on Developmental Disabilities, 7, 123-141.

Drager , K. D. (2009). Aided modeling interventions for children with autism spectrum disorders who require augmentative and alternative communication. Perspectives on Augmentative and Alternative Communication, 18 . 114- 120.
Drager, K. D. R., Postal, V. J., Carrolus, L., Castellano, M., Gagliano, C., \& Glynn, J. (2006). The effect of aided language modeling on symbol comprehension and production in two preschoolers with autism. American Journal of Speech-Language Pathology, 15, 112-125.

Elsabbagh, M., Divan, G., Koh, Y. J., Kim, Y. S., Kauchali, S., Marcín, C., Montiel-Nava, C., Patel, V., Paula, C. S., Wang, C., Yasamy, M. T., \& Fombonne E. (2012). Global Prevalence of Autism and Other Pervasive Developmental Disorders. Autism Research, 5(3), 160-179.

Fagundes, A. (1985). Descrição, definição e registro de comportamentos. São Paulo: Edicon.

Ferreira, P.R., Teixeira, E.V.S., \& Brito, D.B.O. (2011). Relato de Caso: Descrição da evolução da comunicação alternativa na pragmática do adulto portador de autismo. Revista CEFAC: Atualização Científica em Fonoaudiologia e Educação. 13(3), 559-567.

Fidalgo, A. P., Godoi, J.P., \& Gioia, G.S. (2000). Análise de um procedimento de comunicação funcional alternativa (Picture Exchange Communication System). Revista Brasileira de Terapia Comportamental e Cognitiva, 10 (1), 51-66.

Flippin, M., Reska, S., \& Watson, L. R. (2010). Effectiveness of the Picture Exchange Communication System (PECS) on Communication and Speech for Children With Autism Spectrum Disorders: A Meta-Analysis. American Journal of SpeechLanguage Pathology, 19, 178-195.

Frea, W. D., Arnold, C. L., \& Vittimberga, G. L. (2001). A demonstration of the effects of augmentative communication on the extreme aggressive behavior of a child with autism within an integrated preschool setting. Journal of Positive Behavior Interventions, 3, 194-198.

Frost, L. A., \& Bondy, A. S. (2002). PECS: The Picture Exchange Communications System. Training Manual. Pyramid Educational Consultants, Inc.

Ganz, J. B., Simpson, R. L., \& Corbin-Newsome, J. (2007). The impact of the Picture Exchange Communication system on requesting and speech development in preschoolers with autism spectrum disorders and similar characteristics. Research in Autism Spectrum Disorder.

Gomes, R. C. (2011). Interações comunicativas entre uma professora e um aluno com transtorno invasivo do desenvolvimento na escola regular. Dissertação de Mestrado, Universidade Federal do Rio Grande do Norte, Natal-RN.

Kennedy, C.H. (2005). Single-case designs for educational research. Boston: Pearson Education, Inc. 
Klin, A. (2006). Autismo e síndrome de Asperger: uma visão geral. Revista Brasileira de Psiquiatria, 28 (1), 3-11.

Kravits, T., Kamps D. M., Kemmerer K., \& Potucek J. (2002). Brief report: Increasing communication skills for an elementary-aged student with autism using the Picture Exchange Communication System. Journal of Autism and developmental Disorders, 32, 225230.

Macedo, E. C., \& Orsati, F. (2011). Comunicação alternativa. Em J. S. Schwartzman, \& C. A. Araújo (Orgs.), Transtornos do Espectro do Autismo (pp. 244-254). São Paulo: Memnon.

Miura, R. K. K. (2008). Considerações sobre o Currículo Funcional Natural - CFN. Em A. A. S. Oliveira (Org.), Inclusão Escolar: contribuições da Educação Especial (1ª ed., v. 1, pp. 155-165). São Paulo: Cultura Acadêmica Editora.

Mongillo, E. A., Irwin, J. R., Whalen, D. H., Klaiman, C., Carter, A. S., \& Schultz, R. T. (2008). Audiovisual processing in children with and without autism spectrum disorders. Journal of Autism and Developmental Disorders,38, 1349-1358.

National Autism Center (2011). Evidence-Based Practice Autism in the Schools: a guide to providing appropriate interventions to students with autism spectrum disorders. 2. ed. Randolph, Massachusetts: National Autism Center. Recuperado: 23 fev. 2013. Disponível: http://www.nationalautismcenter.org/pdf/NAC\% 20Ed\%20Manual_ FINAL.pdf.

Nunes, D. (2008). AAC Interventions for autism: A research summary. International Journal of Special Education, 23, 17 - 26.

Nunes, D. R. P. (2008). Teoria, pesquisa e prática em Educação: a formação do professor-pesquisador. Educ. Pesqui., 34 (1), São Paulo.

Nunes, D., Azevedo, M. Q. O., \& Freire, J. G. (2011). Comunicação alternativa em sala de aula: relatos de uma professora. Em L. Nunes, M. Pelosi, \& C. Walter (Orgs.), Compartilhando experiências: ampliando a comunicação alternativa (v. 1, pp. 161174). Marília : ABPEE.

Nunes, D., \& Hanline, M. F. (2007). Enhancing the alternative and augmentative communication use of a child with autism through a parent implemented naturalistic intervention. International Journal of Disability, Development and Education, 54, 177-197.

Nunes, D. R. P.; Nunes Sobrinho, F. P. (2010). Comunicação alternativa e ampliada para educandos com autismo: considerações metodológicas. Rev. bras. educ. espec., 16 (2).
Nunes, L. R. P. (1992) Métodos Naturalísticos para o Ensino da Linguagem Funcional em Indivíduos com Necessidades Especiais. Em E.S. Alencar (Org.), Novas Contribuições aos Processos de Ensino e Aprendizagem. São Paulo: Cortez Editora.

Pereira, A., Riesgo, R. S., \& Wagner, M. B. (2008). Autismo infantil: tradução e validação da Childhood Autism Rating Scale para uso no Brasil. Jornal de Pediatria, 84 (6), 487-494.

Preston, D., \& Carter, M. (2009). A review of the efficacy of the Picture Exchange Communication System intervention. Journal of Autism and Developmental Disorders, 39, 1471-1486.

Prizant, B., \& Wetherby, A. (1998). Understanding the continuum of discrete-trial traditional behavioral to social-pragmatic developmental approaches in communication enhancement for young children with autism/PDD. Seminars in Speech and Language, 19, 329-353.

Simpson, R. (2005). Evidence-based practices and students with autism spectrum disorders. Focus on Autism and Other Developmental Disabilities, 20 (3), 140-149.

Sulzer-Azaroff, B., Hoffman, A. O., Horton, C. B., Bondy, A., \& Frost, L. (2009). The Picture Exchange Communication System (PECS): What do the data say? Focus on Autism and Other Developmental Disabilities, 24, 89-103.

Thompson, T. (2011). Individualized Autism Intervention for Young Children: Blending discrete trial and naturalistic strategies. Baltimore, MD: Brookes Publishing Company.

Tien, K. C. (2008). Effectiveness of the Picture Exchange Communication System as a functional communication intervention for individuals with autism spectrum disorders: A practice-based research synthesis. Education and Training in Developmental Disabilities, 43 (1), 61-76.

Walter, C.; Almeida, M. A. (2010) Avaliação de um programa de comunicação alternativa e ampliada para mães de adolescentes com autismo. Rev. bras. educ. espec. [online], vol.16, n.3 [citado 2013-04-15], pp. 429-446

Wendt, O. (2009) Research on the use of graphic symbols and manual signs. Em P. Mirenda, \& T. lacono (Orgs.), Autism Spectrum Disorders and AAC (pp. 83-137). Baltimore: Paul H. Brookes. 


\section{Sobre as autoras}

Débora Regina de Paula Nunes (deboranunes@ufrnet.br)

Professora Doutora do Departamento de Fundamentos e Políticas da Educação da Universidade Federal do Rio Grande do Norte

\section{Larissa Bezerra dos Santos}

Graduanda do Curso de Pedagogia da Universidade Federal do Rio Grande do Norte, Bolsista de Iniciação Científica.

Apoio financeiro - Propesq - UFRN 